\title{
The Use Ex-Vivo Renal Autotransplantation to Treat Hypertension Secondary to Renal Artery Disease: Two Case Reports
}

\author{
Bruno Machado ${ }^{1}$ René Murilo², Reyan Saghir ${ }^{3}$, Noman Saghir ${ }^{4}$, Manoj Poojary ${ }^{5}$, and Marcos Sforza6,* \\ ${ }^{1}$ Assistant Professor, Department of Urology, University of Arkansas, Little Rock, USA \\ ${ }^{2}$ Consultant, Department of Urology, HUCFF-UFRJ, Rio de Janeiro, Brazil \\ ${ }^{3}$ Medical Student, King's College, London, UK \\ ${ }^{4}$ Consultant, Department of Plastic Surgery and Burns, Wythenshawe Hospital, Manchester, UK \\ ${ }^{5}$ Consultant, Department of Anaesthesia, Dolan Park Hospital, UK \\ ${ }^{6}$ Professor, Department of Surgery, SESA and UNFESO universities, Brazil
}

*Corresponding author: Marcos Sforza, Professor, Department of Surgery, SESA and UNFESO Universities, Brazil, E-mail: marcos@marcossforza.com

Received: 10 Mar, 2020 | Accepted: 12 Mar, 2020 | Published: 17 Mar, 2020

Citation: Machado B, Murilo R, Saghir R, Saghir N, Poojary M, et al. (2020) The Use Ex-Vivo Renal Autotransplantation to Treat Hypertension Secondary to Renal Artery Disease: Two Case Reports. J Surg Open Access 6(3): dx.doi.org/10.16966/2470-0991.210

Copyright: (C) 2020 Machado B, et al. This is an open-access article distributed under the terms of the Creative Commons Attribution License, which permits unrestricted use, distribution, and reproduction in any medium, provided the original author and source are credited.

\section{Abstract}

Renal Autotransplantation (RAT) is the surgical procedure in which the kidney is initially removed and subsequently re-implanted in a different position, allowing for improved outcomes in conditions involving ureteral pathology, renovascular and neoplastic disease primarily.

In this paper, we aim to build upon the understanding of RAT and especially its effectiveness in treating patients with hypertension secondary to renal artery disease, intolerant to previous treatment approaches. In particular, the ex-vivo technique will be focused upon as introduced by Ota et al. in 1967 whereby the use of the workbench is frequently applied for patients requiring in excess of 45 minutes of ischaemic time.

We, therefore, put forth two cases managed in co-operation by the University of Arkansas vascular and urology departments. The first of which was a 52-year-old woman with an aneurysmal Lesion reaching the renal artery at the hilum. The second was an 18-year-old woman with Takayasu arteritis. The use of vasopressin had preserved some renal function however at the time of the diagnosis, they were experiencing difficulty in controlling their hypertension, and thus RAT was performed, and the subsequent patient postoperative outcomes and effectiveness have been recorded and analysed as part of this study.

Keywords: Renal autotransplantation; Arterial hypertension; Takayusu arteritis; Renal aneurysms; Bench work

\section{Introduction}

Renal autotransplantation (RAT) is a relatively well-documented practice. The concept was first introduced at the turn of the $20^{\text {th }}$ century when Ullmann and Carrel performed the first RAT on animals. Hardy subsequently undertook the task of performing the first human RAT in the United States. This was followed by Ota, who in 1967 successfully performed the first ex-vivo RAT; a technique whereby the organ is removed and prepared outside of the patient's body $[1,2]$.

The current protocol dictates that Percutaneous Transluminal Angioplasty (PCA) is the optimal treatment method for renal artery stenosis, particularly in isolated occlusive lesions with arteriosclerotic aetiology or myofiber dysplastic lesions. However, the use of PCA may be ineffective or even harmful in patients with extensive renal artery involvement. This is more commonly seen in patients with Takayasu's arteritis, dysplastic myofiber lesions and those with multiple vessel involvement [3]. Several studies have sought to determine the extent of vascular injury required to illicit hypertension, requiring subsequent invasive treatment. They have further demonstrated the absence of the hyperactivation reaction of the renin-angiotensin system in cases with arterial luminal stenosis exceeding $80 \%$. Furthermore, the execution of luminal dilating procedures of the renal arteries has shown to be relatively ineffective [2-10]. RAT offers an effective alternative for those patients with multiple arterial lesions and clinical blood pressure control failure.

\section{Case 1}

The first case was diagnosed with a right renal artery aneurysm located at the bifurcation site of the upper and lower segmental renal arteries.

We present a 52-year-old female of Caucasian descent, who by the means of taking Enalapril and Nifedipine, had reasonably controlled her hypertension, of which she possessed a 15-year history. This 
patient initially presented with complaints of right lower back pain seemingly consistent with osteo-muscular character; pain worsened upon physical exertion and alleviated with rest. Upon the completion of panoramic radiograph imaging of the abdomen, we first noted the presence of a radiopaque image contiguous with the right kidney. The aforesaid image was rounded with further radiopaque boundaries containing centrally radiotransparent areas (Figure 1).

Abdominal computed tomography scans allowed us to further visualise this area, revealing fusiform dilatation and arterial wall calcification of the right renal artery (Figure 2).

The patient was also subjected to aortorenal angiogram, with a specific study of the renal arteries to display an aneurysm of the right renal artery. It showed an elongated fusiform aspect that affected the bifurcation of the upper and lower segmental renal arteries.

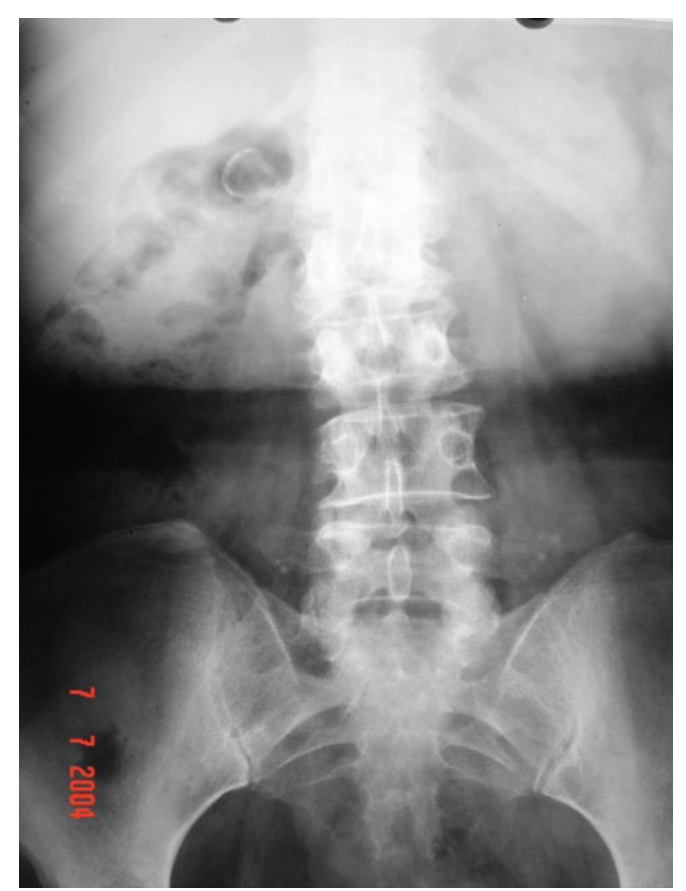

Figure 1: Abdominal X-Ray.

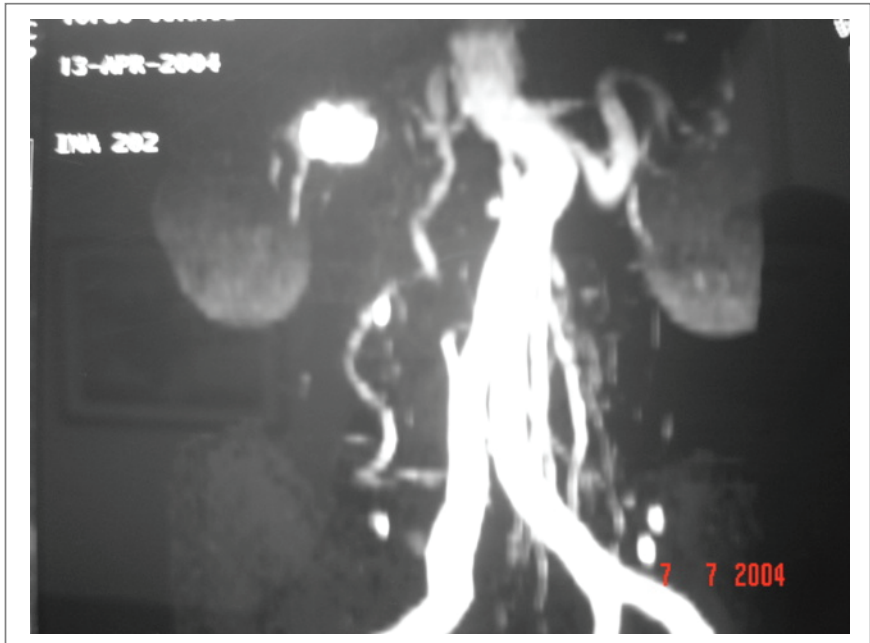

Figure 2: Abdominal CT scan Reconstruction.
Surgical intervention, more specifically, a right lobotomy for nephrectomy with careful preservation of the renal hilar vessels and periureteral fat envelope were performed upon this patient.

Preparation of the kidney was performed ex vivo, in addition to a 1 liter Collins solution infusion. In order to maintain the body at lower temperatures, the infusion had been previously cooled to $4^{\circ} \mathrm{C}$ and was thereupon placed in a vat filled with an equally cooled saline solution, at $4^{\circ} \mathrm{C}$.

We performed the excision of the aneurysm and repair of the defect in the wall of the renal artery with patches. (Figures 3 and 4). After the treatment of arterial injury, the kidney was implanted into the right iliac fossa retroperitoneal in position.

The revascularization of the kidney was performed with a terminoterminal anastomosis of the right renal artery in the right internal iliac artery, and end-to-side anastomosis of the right renal vein with the right external iliac vein, both with the usages of 6.0 polypropylene sutures.

A "double J" ureter catheter was placed and anastomosed end-toend with its distal stump using polydioxanone 6.0 wires. The site of surgery was drained with a Penrose drain. In total, the surgery lasted approximately four hours.

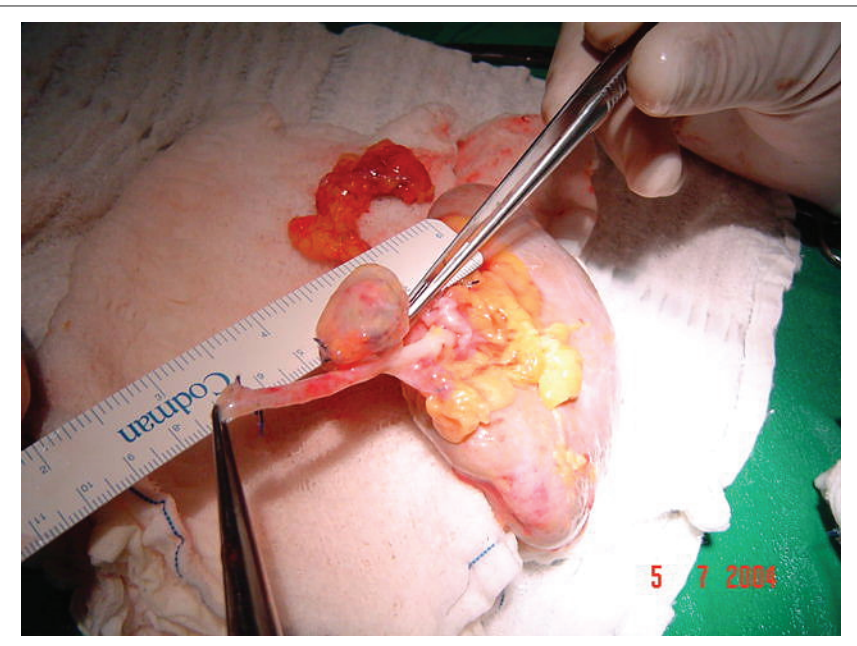

Figure 3: "Bench Work" Renal Artery Aneurism.

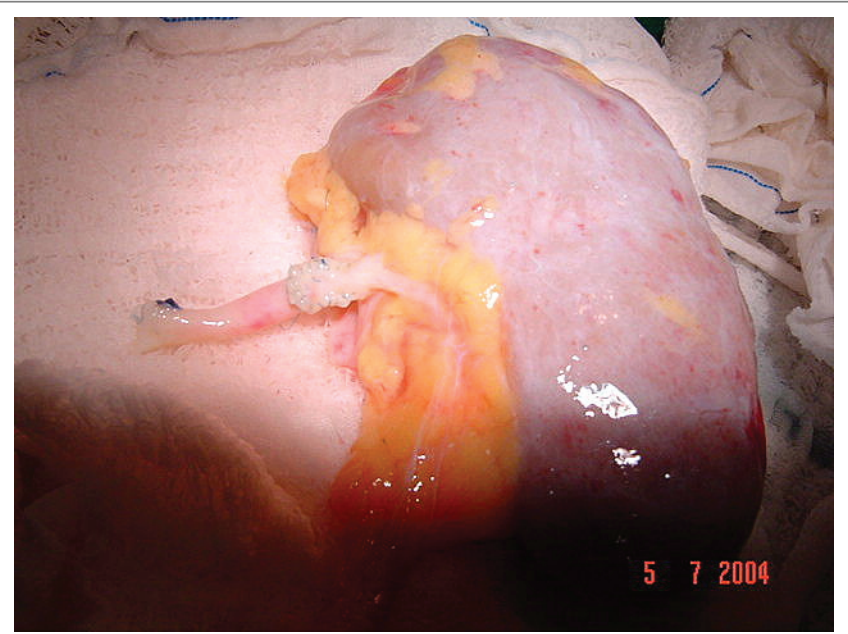

Figure 4: "Bench Work" Renal Artery Repair. 
The double-J catheter was removed four weeks after surgery as an outpatient.

\section{Case 2}

Our patient in case 2 had Takayasu's disease type II, confirmed by criteria guidelines set out by Ueno-Lupi-Herrera $[8,9]$. The anatomical variations present in our patient were aneurysmal dilatations of the descending aorta between; he emergence of the subclavian artery to the thoracoabdominal junction, and the abdominal portion of the aortic coarctation until the presentation of the renal arteries.

We present an 18-year-old female patient of mixed-race ethnicity, suffering from hypertension, weight loss, and dyspnoea at rest. Additionally, she had also been experiencing episodes of paroxysmal nocturnal dyspnoea, orthopnoea and intermittent claudication.

The clinical picture originated five months before our patients' initial attempt to seek medical assistance, she had been using: Atenolol, Digoxin, Methyldopa, and Hydrochlorothiazide. Computerized angiography imaging of the chest and abdomen brought a pair of aortic aneurysms to our attention. The first, spanning from the emergence of the subclavian artery to the thoracoabdominal junction, and the second arising from the abdominal portion of aortic coarctation, until the emergence of the renal arteries.

The surgical approach for this patient was assumed through a swordtail-pubic incision for the repair of various vascular lesions, followed by a bilateral nephrectomy with the preservation of the renal hilar vessels and the fatty periureteral envelope. During the procedure, we noted the comparatively small size of the left kidney including its respective hilar vessels and small arms. After the bilateral nephrectomy, the kidneys were prepared ex vivo, with the infusion of 1 liter of Collins solution cooled to $4^{\circ} \mathrm{C}$ and placed in a tub with cooled saline solution, in order to maintain the bodies at $4^{\circ} \mathrm{C}$.

Implantation of the left kidney was fixed in an orthotopic position, with the renal artery anastomosed in termino-terminal position of the splenic artery, renal vein and the inferior mesenteric vein (Figure 5).

Thrombosis of the left renal artery anastomosis was diagnosed intraoperatively. By the use of a Fogarty catheter, we made an attempt at balloon embolectomy, which proved to be unsuccessful. It was then decided upon extraction of the left kidney, that all vascular anastomoses were to be performed with 6.0 polypropylene sutures.

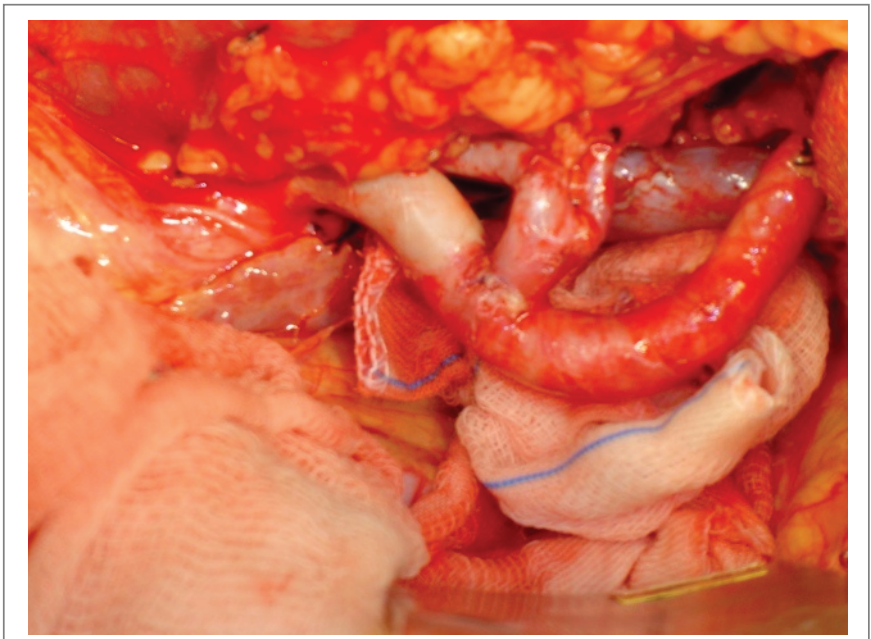

Figure 5: Renal Vessels anatomosis (in vivo).
The right renal vessels were anastomosed end-to-terminally in the external iliac vessels, again performed with 6.0 polypropylene sutures.

Within the ureter, we inserted a "double J stent" which was anastomosed end to end with its distal stump using polydioxanone 6.0 wires. The surgical site was drained with a Penrose drain. In total, the surgery lasted approximately ten hours. Four weeks following the surgery, the double-J catheter was removed as an outpatient. The average ischemic time in each kidney in both cases was 1 hour and 20 minutes.

\section{Discussion}

RAT can be useful for multiple indications as outlined in table 1 and demonstrated by both our cases above. Our patients had two different diseases however they had both progressed to uncontrollable hypertension, caused by multiple changes in the caliber of the renal arteries (Table 1).

Two years after their respective surgeries, we assessed both patients via telephone contact by asking a set of closed questions, prepared by simplifying several questionnaires in the literature shown in table 2 $[2-4,8,9]$. The questionnaire was also designed according to the criteria defined by de Góes GM, et al. [9]. After analysis of the questionnaire, we observed that there was a high degree of patient satisfaction with the procedure and that there was a significant improvement when compared with data from the preoperative use of antihypertensive medications (Table 2).

Table 3 demonstrates the results of autotransplants, as quoted by two authors, in their series. Our series is consistent with the results from Góes GM, et al. [9] and Chiche L, et al. [4] corroborating the results of the review of the literature cases (Table 3 ).

The choice of technique performed was ex vivo as several authors have shown that when there is a need for complex vascular repair surgery in vivo, it leads to kidney ischemia exceptionally faster, the failure to maintain proper perfusion and adequate cooling is what

Table 1: Indications Auto-kidney transplant.

1. Renal artery stenosis by Fibromyodisplasia (FMD) and atherosclerotic plaques.

2. Vascular injury or ureteral complex.

3. O-Venous Fistula Artery.

4. Takayasu arteritis

5. Low back pain-hematuria syndrome.

6. Renal tumors or urethral.

7. Tuberculosis, Renal or Urethral.

8. latrogenic urethral injuries.

9. Extensive ureteral strictures.

10. Calculations.

11. Retro-peritoneal fibrosis.

Adapted from Wotkowicz C, et al. [2]; Plas E, et al. [8]

Table 2: Telephone responses to the questionnaire.

\begin{tabular}{|l|c|c|}
\hline \multicolumn{1}{|c|}{ Questions } & Patient $\mathbf{0 1}$ & Patient $\mathbf{0 2}$ \\
\hline Feel-better after the procedure? & Yes & Yes \\
\hline Use antihypertensive medication? & Yes & Not \\
\hline How many medications are used? & 01 & No \\
\hline Satisfied with the surgery? & Yes & Yes \\
\hline
\end{tabular}

Source: Department of Urology; HUCFF-UFRJ 
Table 3: Results of renal autotransplantation in the levels of PA in the literature.

\begin{tabular}{|l|c|c|c|c|c|}
\hline & \multicolumn{2}{|c|}{ de Góes GM, et al. [9] } & \multicolumn{3}{c|}{ Chiche L, et al. [4] } \\
\hline & FMD* & Arteriosclerosis & FMD* & Arteriosclerosis & Takayasu \\
\hline Cured & $75 \%$ & $0 \%$ & $96 \%$ & $50 \%$ & $89 \%$ \\
\hline Improved & $12.5 \%$ & $60 \%$ & & & $12 \%$ \\
\hline No change & $12.5 \%$ & $40 \%$ & $3.8 \%$ & $30 \%$ & $0 \%$ \\
\hline Cured & $0 \%$ & $0 \%$ & $0 \%$ & $10 \%$ & \\
\hline
\end{tabular}

*Fibromyodisplasia

Table 4: "Guidelines" for surgery bench $[3,11]$.

1. Surgical time greater than 45 minutes.

2. Need to distal dissection of the renal vasculature and thin.

3. Reno-vascular disease refractory to treatment with ATP.

4. Distal stenosis in the arteries is reindeer.

5. Intrarenal aneurysms.

6. Arteriovenous malformations.

7. Multiple vascular disease.

8. Ureteral injuries and calculosas refractory to conventional treatments

9. Renal cell carcinoma in solitary kidney patients depending on their anatomical location and history.

Adapted from: Wotkowicz C, et al. [2]; Knobloch K, et al. [7].

ultimately limits the surgical time window $[2-4,6,7,9]$. The constitution of a "bench work", following the guidelines listed in table 4, allowed surgeons to work in a calmer and ruled fashion, contributing to the success of clinical outcome as seen in our cases $[2-5,8,9,11]$ (Table 4 ).

\section{Conclusion}

Our experience confirmed what the literature shows as valid: RAT is a safe technique with positive postoperative outcomes. The ex vivo surgery improves the surgical technique and allows more complex vascular repairs to be performed more accurately, without having to worry about the ischemic time. In selected cases, RAT presents itself as a viable option for the treatment of hypertension secondary to renal arterial disease.

\section{Consent}

Written informed consent was obtained from the patient for publication of this case report and accompanying images. A copy of the written consent is available for review by the Editor-in-Chief of this journal.

\section{Competing Interest}

The authors declare that they have no competing interests.

\section{References}

1. Nagy J (1999) A note on the early history of renal transplantation: Emerich (Imre) Ullmann. Am J Nephrol 19: 346-349.

2. Wotkowicz C, Libertino JA (2004) Renal autotransplantation. BJU Int 93: 253-257.

3. Cho CS, Robinson PW, Grant AB, Hibberd AD (2001) Successful ex vivo renal artery reconstruction and renal autotransplantation. ANZ J Surg 71: 79-82.

4. Chiche L, Kieffer E, Sabatier J, Colau A, Koskas F, et al. (2003) Renal autotransplantation for vascular disease: late outcome according to etiology. J Vasc Surg 37: 353-361.

5. Hahn D, Thomson PD, Kala U, Beale PG, Levin SE (1998) A review of Takayasu's arteritis in children in Gauteng, South Africa. Pediatr Nephrol 12: 668-675.

6. Martínez JCE, Rojas LRL, Sarduy JDB, Diaz JH (1996) Diagnóstico clínico y angiográfico de la arteritis de Takayasu. Rev Cubana Cardiol Cir Cardiovasc.

7. Knobloch K, Wiebe K, Licntenberg A, Fischer S, Gohrbandt B, et al. (2005) Ex Vivo Repair and Renal Autotransplantation for Complex Renal Artery Aneurysms in a Solitary Kidney. Ann Vasc Surg 19: 407410.

8. Plas E, Kretschmer G, Stackl W, Steininger R, Mühlhacher F, et al. (1996) Experience in renal autotransplantation: analysis of a clinical series. Br J Urol 77: 518-523.

9. de Góes GM, Lucon AM (1981) Renal Autotransplantation in the Treatment of Hypertensive Disease Associated with Unilateral Renal Artery Stenosis. J Urol 126: 14-16.

10. Simon $\mathrm{G}$ (2000) What is critical renal artery stenosis? Implications for treatment. Am J Hypertens 13: 1189-1193.

11. Retik W, Wein V (2002) Renal Transplantation. In: Campbell MF, Walsh PC, Retik AB (eds) Campbell's Urology. $8^{\text {th }}$ edition, The University of Michigan, Saunders 345-376. 